\title{
CHARACTERIZATION AND COIL TEST RESULTS FOR A MULTIFILAMENTARY NbTi CONDUCTOR UTILIZING ARTIFICIAL PINNING CENTER TECHNOLOGY
}

\author{
R.M.Scanlan, D.R.Dietderich, P.McManaman, and W.Ghiorso \\ Lawrence Berkeley Laboratory \\ 1 Cyclotron Road \\ Berkeley, CA 94720
}

\begin{abstract}
The introduction of pinning centers via the controlled addition of a second phase, with the correct size and spacing, has been proposed as a method for producing a material with optimum flux pinning and hence a higher critical current density in practical superconductors. The demonstration of such artificial pinning center (APC) materials has been the aim of recent collaborative efforts with several U.S. manufacturers. This paper reports the coil test results for a multifilamentary $\mathrm{NbTi}$ conductor fabricated using an APC technique. The conductor showed improved performance compared to earlier APC conductors, and its performance is comparable to that found in conventional multifilamentary $\mathrm{NbTi}$ conductors. In addition to coil test results, the paper will report on the low field magnetization, SEM, and TEM studies, and the results will be compared with similar results on conventional NbTi.
\end{abstract}

\section{INTRODUCTION}

The concept of applying an artificially produced array of defects to act as flux pinning centers has been proposed many times as a method for studying flux pinning under more controlled conditions than can be achieved by relying on precipitation or cold work. An example of these earlier approaches includes the production of multilayer thin film structures (1). Interest in APC as a method for producing practical superconducting materials was stimulated by a report in 1985 (2), which not only presented some theoretical estimates of potential Jc values for practical superconductors but demonstrated that very good Jc values can be achieved, under some conditions, in $\mathrm{NbTi}$ with artificial pinning centers. In the U.S., APC studies were initiated by Intermagnetics General Corp. (IGC), and they were granted a patent for their approach in 1989 (3). Shortly thereafter, the present authors began collaborating with several U.S. superconductor manufacturers to explore the possibility of producing a practical superconductor based on the APC approach. Scale-upefforts were funded at IGC, Teledyne Wah Chang Albany (TWCA), and Supercon in order to answer the following questions: (1) Can APC superconductors reach and hopefully exceed the Jc values achieved in commercially available superconductors?

This work was supported by the Director, Office of Energy Research, Office of High Energy and Nuclear Physics, Division of High Energy Physics of the U.S. Department of Energy under Contract No. DE-AC03-76SF00098.
(2) Can these high Jc values be maintained at high fields? and (3) Can these conductors be produced in a cost effective manner? The first question was answered in the affirmative by the work at IGC $(4,5)$, which produced conductors with Jc values exceeding those for commercially available $\mathrm{NbTi}$ at low fields, and comparable to the commercially available conductors at $5 \mathrm{~T}$. However, the results were not encouraging with regard to the other two goals. The Jc values decreased rapidly in fields above $5 \mathrm{~T}$, so that at $7 \mathrm{~T}$ the best values were only about $60 \%$ of that available in commercially available $\mathrm{NbTi}$ wires. Also, the manufacturing process required one extra extrusion step in order to prepare the monofilament which contained the APC structure; this extra step reduces the overall process yield and increases the cost of the process.

In order to achieve the goals of better high field performance and reduced manufacturing cost. while maintaining the high Jc performance, additional work was initiated with other manufacturers. TWCA proposed a jelly roll method for fabricating the monofilament containing the APC structure, thus eliminating the extra extrusion step. Supercon proposed an approach that would, if successful, both improve the high field performance of the APC conductor and reduce its cost. Both the TWCA approach and the Supercon approach were evaluated through the initial phase of fabricating samples and measuring critical current densities. At the end of this phase, the Supercon approach was chosen for further scale-up. This paper describes the results of this scale-up program, which has resulted in the first demonstration of an APC superconductor in a practical device.

\section{EXPERIMENTAL DETAILS}

The goal of this program is to demonstrate the performance of APC NbTi relative to conventional $\mathrm{NbTi}$, to develop an understanding of the role of processing parameters in the development of the APC microstructure, and to begin the process scale-up necessary for the production of useful quantities of this new material. The device chosen for this first demonstration is a solenoid with the parameters listed in Table 1. This solenoid requires a significant length of wire and hence demonstrates the manufacturing scale-up potential. It also operates at a relatively high field, and thus demonstrates the high field capability of the APC material. 
Table 1

\begin{tabular}{|c|c|}
\hline \multicolumn{2}{|c|}{ Solenoid parameters } \\
\hline Design field & $8.0 \mathrm{~T}$ at $230 \mathrm{amps}$ \\
\hline Field & \\
\hline uniformity & $0.2 \%$ over $20 \mathrm{~mm}$ DSV \\
\hline Clear bore & $45 \mathrm{~mm}$ \\
\hline Outside Dim. & $140 \mathrm{~mm}$ diam $\times 203 \mathrm{~mm}$ long \\
\hline Winding pack & 164 turns by 38 layers \\
\hline \multicolumn{2}{|c|}{ Resistance at $300 \mathrm{~K}=46.5 \mathrm{ohms}$} \\
\hline Coil construction & $\begin{array}{l}\text { Vacuum epoxy impregna- } \\
\text { tion }\end{array}$ \\
\hline $\begin{array}{l}\text { Coil operating } \\
\text { mode }\end{array}$ & Persistent current \\
\hline
\end{tabular}

The conductor parameters are listed in Table 2. These parameters were chosen to demonstrate that relatively fine filaments and a high current density can be achieved in a wire with a reasonable diameter and copper to superconductor ratio.

Table 2

\begin{tabular}{|lc|}
\hline & Wire parameters \\
\hline $\mathrm{J}_{\mathrm{c}}(5 \mathrm{~T}, 4.2 \mathrm{~K})$ & $2445 \mathrm{~A} / \mathrm{mm}^{2}$ \\
Diameter & $1.14 \mathrm{~mm}$ uninsulated; \\
& $1.23 \mathrm{~mm}$ insulated \\
Cu to super- & \\
conductor ratio & $1.92 \pm .06$ \\
Filament number & 2994 \\
Filament & 12 microns \\
diameter & $13 \mathrm{~mm}$ \\
Twist pitch & \\
Continuous length & \\
supplied & $2475 \mathrm{~m}$ \\
\hline
\end{tabular}

\section{WIRE MANUFACTURE AND MICROSTRUCTURE}

The wire was produced by Supercon, Inc. using a proprietary process for fabricating the APC type structure. The wire was produced in one piece with a finished length of 2500 meters, insulated with Formvar, and sent to LBL for evaluation and coil construction.

A transverse section of the wire is shown in Figure 1, and an enlarged view of the individual filaments is shown in Figure 2.

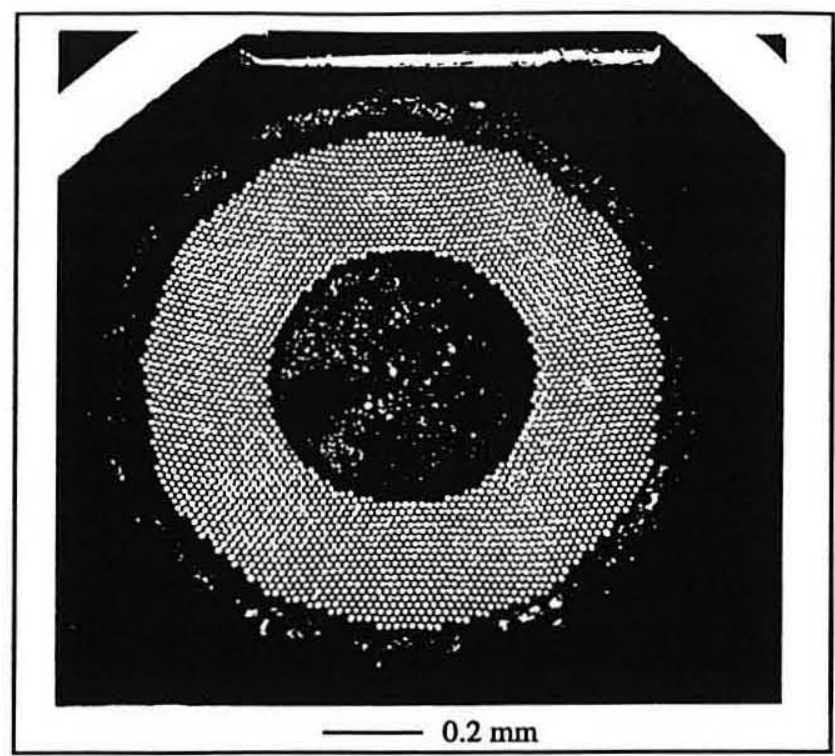

Figure 1. Transverse section of wire at final size with 2994 filaments in a copper matrix.

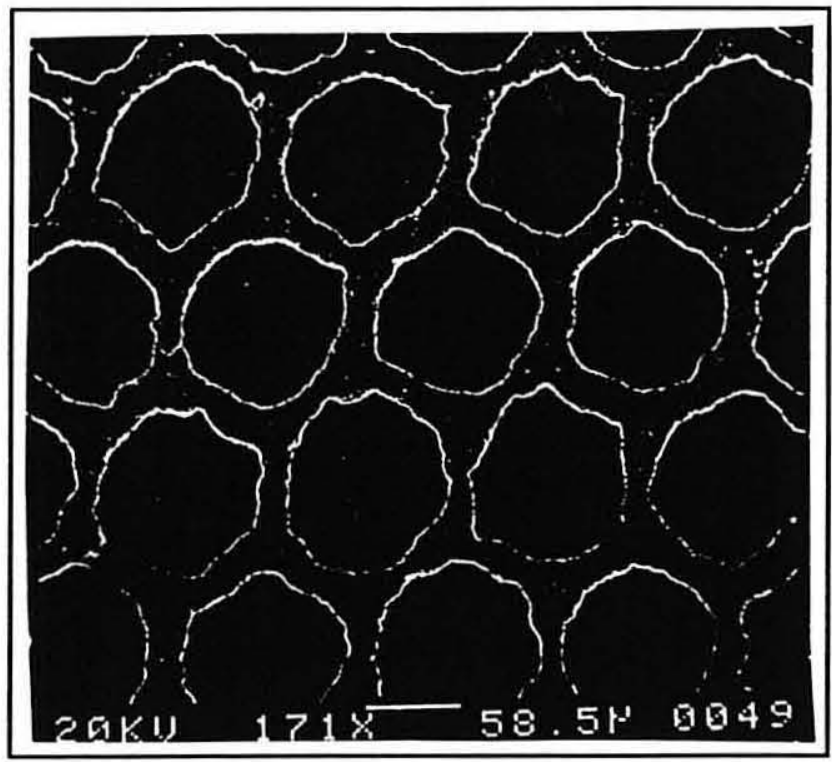

Figure 2 . Enlarged view of filament area at an intermediate stage in processing.

Filament area analysis was performed and the results compared with typical SSC type wire. The standard deviation, normalized to the filament diameter, is $8.6 \%$ for the APC wire at a diameter of $9 \mathrm{~mm}$. and $11.5 \%$ at the final wire diameter of $1.23 \mathrm{~mm}$. These values are to be compared with atypical value of about $8 \%$ for SSC wire (6). Other APC wire made by the same process showed a $5 \%$ area variation in filaments of 16 micron diameter. The somewhat high value for filament size variation in the APC wire used in this coil is believed to be due to extrinsic factors (filament spacing, extrusion conditions) and not to any intrinsic property of the APC wire. The local area ratio (copper:filament) is about 0.6 , which can lead to sausaging in conventional NbTi composites (7). 
The microstructural evolution was followed by SEM observations until the wire size was about $9 \mathrm{~mm}$; the filament circumference stayed uniform to this point, but the individual layers became highly non-uniform, as shown in Figure 3 . The microstructure was also examined at final wire size by TEM, and a typical transverse section is shown in Figure 4. This microstructure appears qualitatively similar to that obtained in the IGC APC material (5); quantitative analysis is still in progress at this time. The structure is composed of a fine ribbon-like pattern. The ribbons are 10-20 nanometers in thickness, and some ribbons appear to contain a substructure of about 1-2 nanometers in thickness. This structure is still being analyzed and the results will be published at a later time.

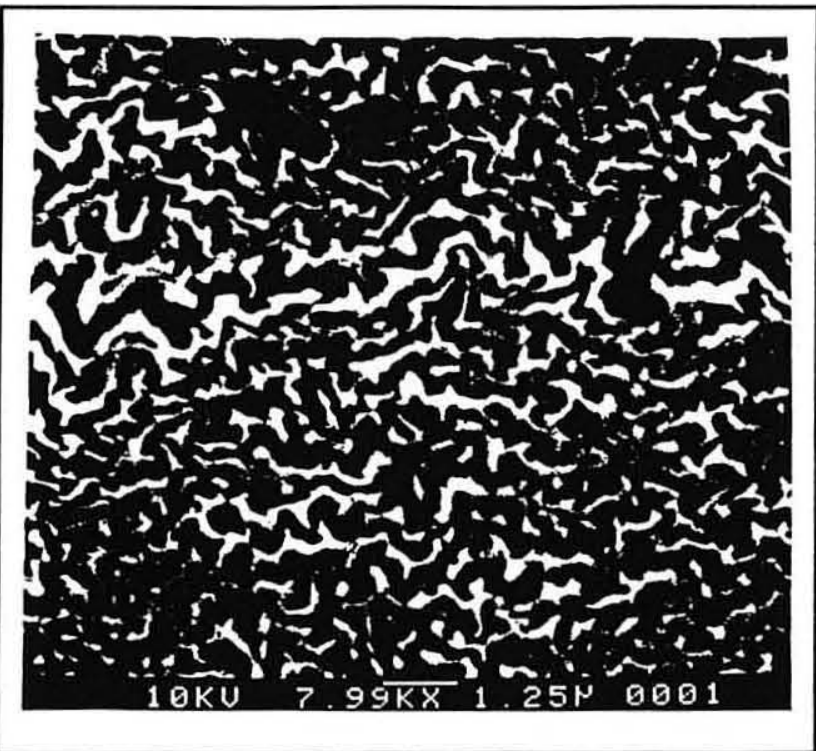

Figure 3 . Transverse section through one filament showing the layers which are highly distorted at this point in the processing.

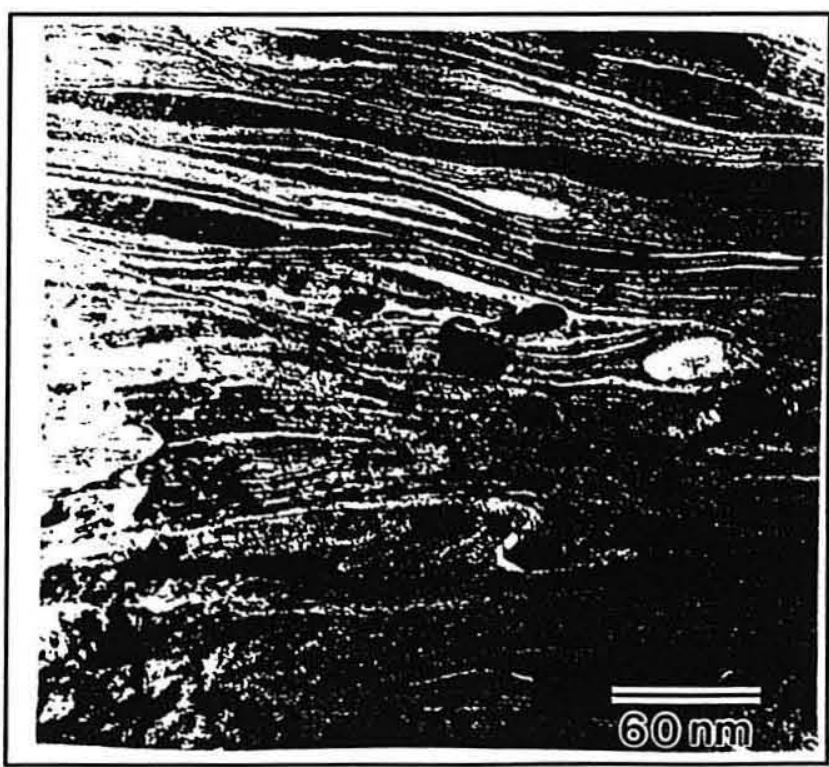

Figure 4 . Bright field transmission micrograph of transverse section of wire after rolling into a tape showing the ribbon-like nature of the microstructure.

\section{COIL CONSTRUCTION AND TESTING}

The coil was wound on a stainless steel bobbin with fiberglass/epoxy insulation applied to the end flanges and the bore tube. The insulation on the end flanges contained radial grooves $1.6 \mathrm{~mm}$ thick by $3.2 \mathrm{~mm}$ wide, 30 degrees apart. A layer-winding approach was used to wind the coil. After the coil was wound and vacuum epoxy impregnated, the persistent current joints were fabricated by Wang NMR, Inc.

The coil was then fitted with a Hall effect field probe and installed in the test cryostat (Figure 5).

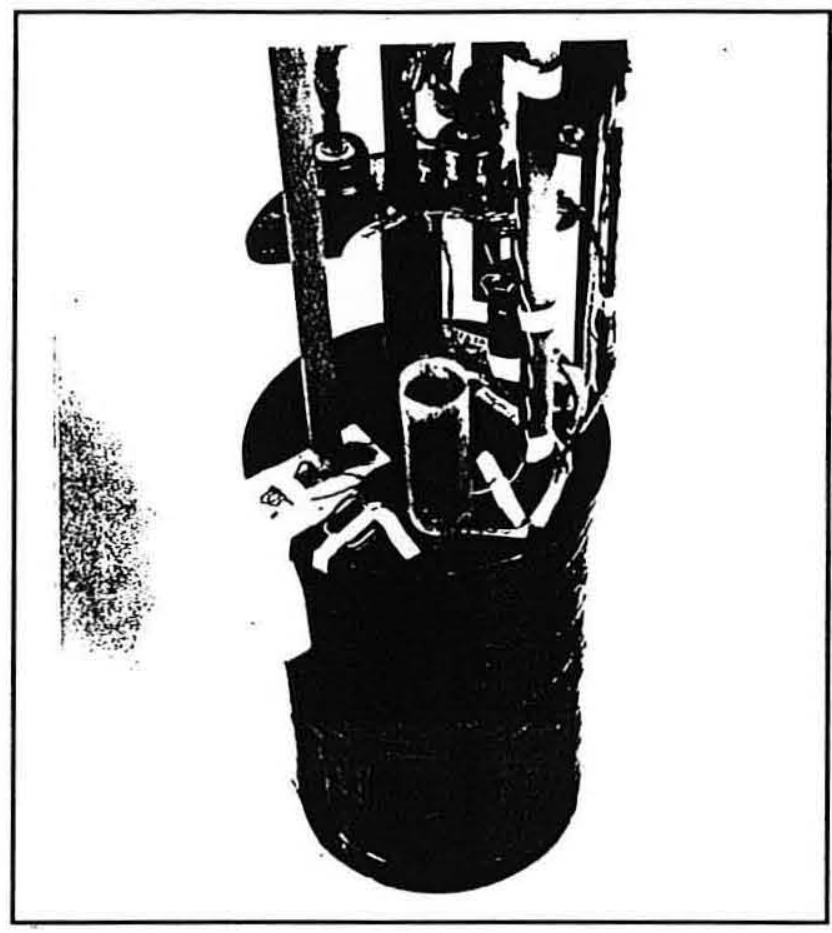

Figure 5. Completed solenoid ready for testing, showing the persistent current joints and outer surface of the epoxy-impregnated coil

The magnet was ramped up to the design current with 0.6 volts maintained at the magnet. This initial charging was completed without any quenches. Next, the persistent current operation was tested at 100,200 , and 230 amps. It was easy to lock into the persistent mode operation, and no decay was noted for the nominal $1 \mathrm{hr}$. hold time. The magnet was allowed to warm to ambient temperature and then recooled for a subsequent test. In this test, the magnet was ramped up until it quenched at a current of $245 \mathrm{amps}$, which corresponds to a field of $8.5 \mathrm{~T}$. This field level was verified by the Hall effect probe which is installed in the magnet bore. This current is $4 \%$ above the measured short sample value, using a $10^{-14} \mathrm{ohm}-\mathrm{m}$ criterion. The magnet load line, together with the short sample performance, is shown in Figure 6. 


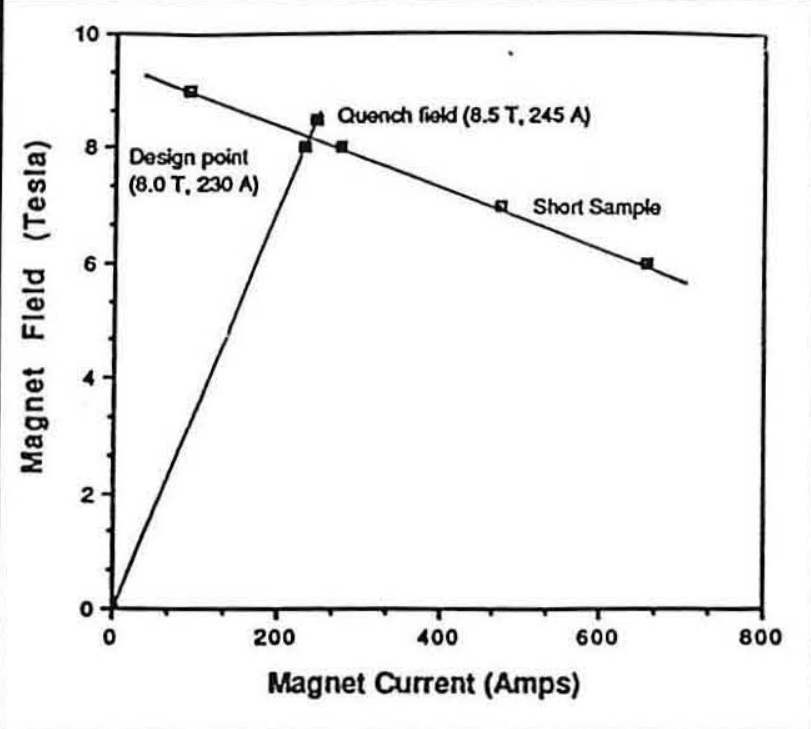

Figure 6. Current vs. field plot for the wire and for the solenoid, operated at $4.2 \mathrm{~K}$. The magnet reached a field slightly above the predicted short sample behavior, without any training quenches.

This magnet has been in operation as a short sample test facility during the period from Oct 1991 to the present, and it has shown very consistent behavior during this time. Nothing in the operating characteristics indicate that the material is made by the APC approach rather than the conventional approach for $\mathrm{NbTi}$.

Since fabrication of this early conductor, Supercon has fabricated inner and outer conductor in sufficient quantity to produce a 1 meter dipole magnet, the first to be made from APC material. The wire has been received by LBL and is being cabled at the present time. Further development of APC conductor design and processing is proceeding at Supercon.

\section{CONCLUSIONS}

1. This solenoid, which represents the first practical device constructed from NbTi wire using the APC approach, performed as expected. The magnet can be easily operated in the persistent current mode.

2. The filament pattern and filament uniformity for the APC material is comparable to currently available SSC and MRI conductors.

3. The APC microstructure consists of a ribbon type structure which is qualitatively similar to that observed in conventionalNbTi. Quantitative analysis stillinprogress.

\section{ACKNOWLEDGMENTS}

We wish to thank our colleagues at Supercon Inc., especially Jim Wong and Mark Rudziak, for the perseverance, dedication and creativity they have shown in producing the wire for this project.

\section{REFERENCES}

1. R.H. Hammond, B.E. Jacobson, T.H. Geballe, J. Talvacchio, J.R. Salem, H.C. Pohl, and A.I. Braginski, IEEE Trans. on Magnetics, Mag-15, p. 619, 1979.

2. G.L. Dorofejev, E.Yu. Klimenko, S.V. Frolov, E.V. Nikulenkov, E.I. Plashkin, N.I. Salunin, and V.Ya. Filkin, Broc. Ninth Intl. Conf. on Magnet Technology, C. Marinucci, P. Weymuth, eds., p. 564, 1985.

3. U.S.Patent $4,803,310$.

4. H.C. Kanithi, P. Valaris, L.R. Motowidlo, B. Zeitlin, and R.M. Scanlan, Advances in Cryogenic Engineering (Materials) 38, p. 675, 1992.

5. D.R. Dietderich, S. Eylon, and R.M. Scanlan, Ibid., p. 685 .

6. J.M. Seuntjens, D.W. Capone II, and W.H. Warnes, Supercollider 3, 1991.

7. E. Gregory, T.S. Kreilick, A.K. Ghosh, and W.B. Sampson, Cryogenics 27, p. 178, 1987. 